\title{
Protocol Deviation Sponsor Defined Identifier
}

National Cancer Institute

\section{Source}

National Cancer Institute. Protocol Deviation Sponsor Defined Identifier. NCI Thesaurus.

Code C83077.

One or more sponsor-defined characters used to identify, name, or characterize the protocol deviations. 